\title{
Chronic Intestinal Idiopathic Pseudo-obstruction (CIIPO) Complicated by Sigmoid Volvulus
}

\author{
Christos Konstantinidis* \\ Surgical Department, Hippocratio General Hospital of Athens, 114 Vasilissis Sofias str, Athens, Region of Attica, Greece \\ *Corresponding author: drchriskons@yahoo.gr
}

\begin{abstract}
A 17-year-old male patient with chronic intestinal idiopathic pseudo-obstruction was admitted to the emergency department clinically deteriorated due to sigmoid volvulus. Initial resuscitative efforts followed by endoscopy failed to detorse and decompress the bowel. At the followed emergency laparotomy the volvulus was derotated and a Hartmann's procedure with left hemicolectomy was performed. The patient after 3 months underwent stoma closure. At one-year follow-up the patient showed clinical improvement.
\end{abstract}

Keywords: constipation, megacolon, pseudo obstruction, sigmoid volvulus

Cite This Article: Christos Konstantinidis, "Chronic Intestinal Idiopathic Pseudo-obstruction (CIIPO) Complicated by Sigmoid Volvulus." American Journal of Medical Case Reports, vol. 5, no. 1 (2017): 22-24. doi: 10.12691/ajmcr-5-1-6.

\section{Introduction}

Intestinal pseudo-obstruction (megacolon) refers to bowel dilation without known mechanical occlusion of the lumen. It can be acute (AIPO), as in Ogilvie's syndrome or chronic (CIPO), either primary or secondary (electrolyte disorders, viruses, toxic causes etc). Primary CIPO, can be congenital or acquired, due to known histopathological abnormalities: neuropathies (intrinsic and extrinsic gastrointestinal nerve pathways affected), mesenchymopathies (interstitial cells of Cajal affected) and myopathies (smooth muscle cells affected) [1].

Chronic intestinal idiopathic pseudo-obstruction (CIIPO) describes permanently distended bowel of unknown origin. In most cases CIIPO is sporadic, while familial forms (autosomal dominant, autosomal recessive and X-linked transmission) have also been described $[2,3]$.

Herein, it is presented a case of sigmoid volvulus in an adult with known CIIPO.

\section{Case Presentation}

A 17-year-old male patient presented to the emergency department with marked abdominal distention, vomiting and referred faecal incontinence for several days. From his medical history he had had mental retardation and chronic constipation since he was one year old. He was receiving long-term laxatives for the treatment of constipation. He has been diagnosed as having non-Hirschsprung congenital megacolon, after mentioned thorough investigation in the past.

On admission his general clinical condition was bad. The patient was lethargic, dehydrated with low blood pressure and tachycardia, tachypneic but afebrile. On clinical examination the abdomen was diffusely distended and tympanic on percussion, with no sign of peritonitis.

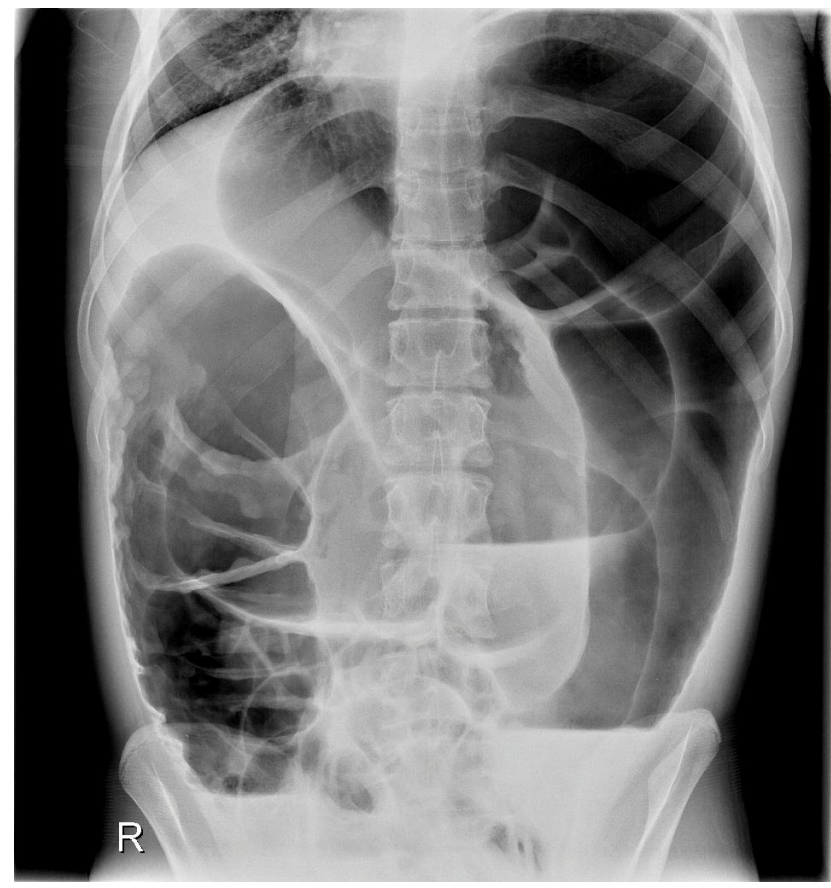

Figure 1. Plain abdominal $x$-ray indicating dilated bowel loops

Plain abdominal radiograph (Figure 1) showed grossly dilated intestinal loops. Subsequent abdominal scan (Figure 2, Figure 3) indicated sigmoid volvulus. Initially, the patient was resuscitated with intravenous fluids while gastric decompression attempted via nasogastric tube. Flexible sigmoidoscopy in the aim of intestinal detorsion and decompression via was unsuccessful. Due to patient's clinical deterioration, he underwent emergency laparotomy, at which massive dilation of bowel loops were seen (Figure 4). There were no signs of bowel ischemia or 
perforation. The sigmoid colon was derotated and left hemicolectomy through Hartmann's procedure was performed. Also, an accidental splenectomy was occurred. Postoperative period was uneventful and the patient was discharged 2 weeks after admission. He was referred to a specialist center for cytogenetic (chromosomal) analysis, which didn't reveal morphological anomalies of autosomal or X-linked chromosomes. Histopathological examination of the resected specimen was not indicative of Hirschsprung's disease.

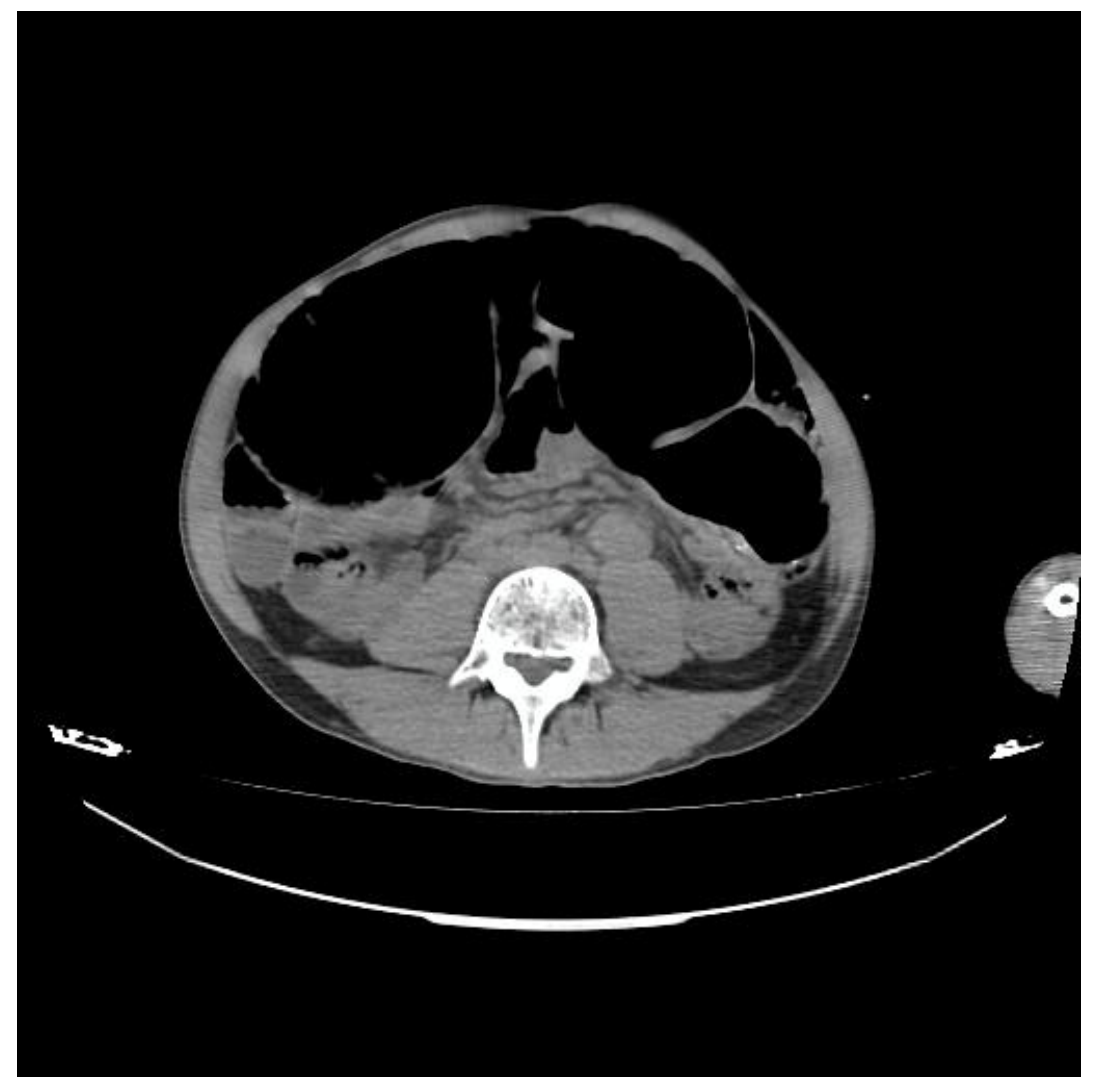

Figure 2. Abdominal scan indicating sigmoid volvulus

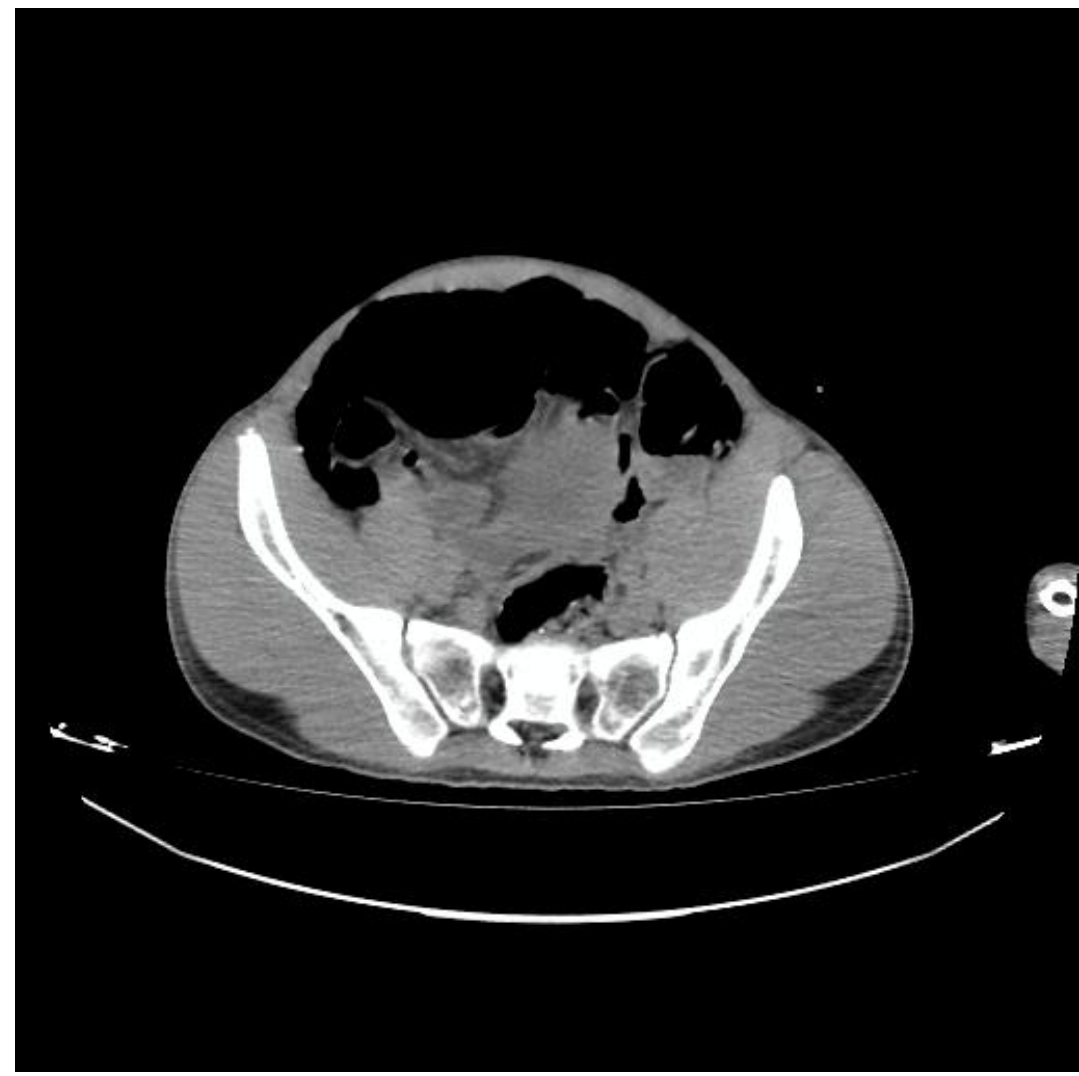

Figure 3. Another abdominal scan view indicating sigmoid volvulus 


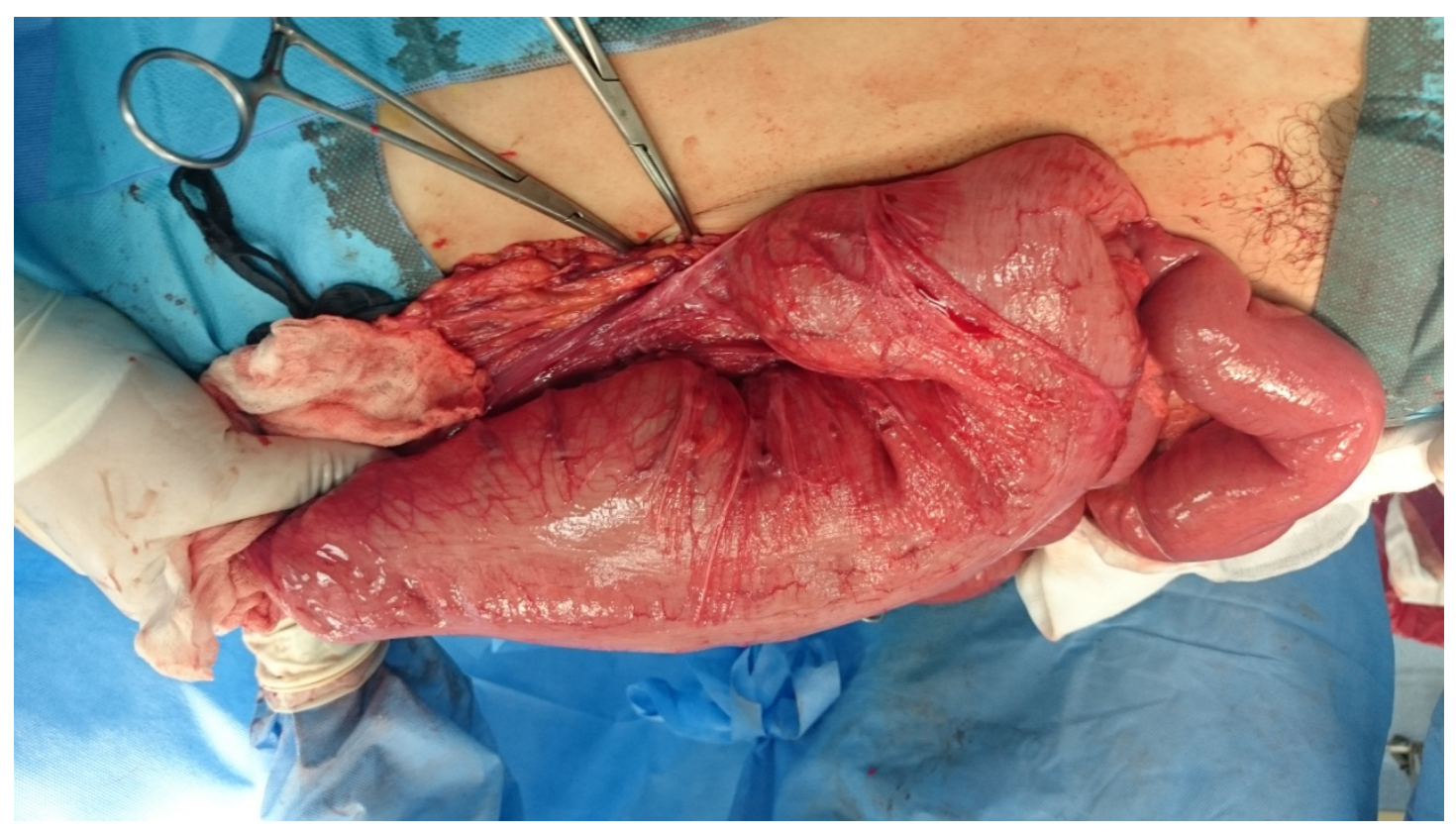

Figure 4. Laparotomy image showing gross dilation of the descending and sigmoid colon

Stoma closure was performed 3 months postoperatively. At one-year follow-up the patient is in good condition, with gained weight and less requirements for laxatives.

\section{Discussion}

Among large bowel, sigmoid colon is more often affected from volvulus and is more frequent in adults [4]. Initial colonoscopic attempt for decompression and detorsion of the affected bowel segment is the treatment of choice, when it is possible and the clinical situation of the patient allows it. In suspicious or obvious peritonitis, as well as when the patient deteriorates, urgent laparotomy is indicated $[5,6]$. While Hirschsprung's disease represents the most known form of congenital megacolon, affecting from distal part of sigmoid colon and rectum to total large bowel [7], chronic intestinal pseudo-obstruction (CIPO) is a rare syndrome, with a particular subgroup called chronic idiopathic intestinal pseudo-obstruction (CIIPO) [8]. The latter characterizes chronic constipation accompanied with persistent bowel dilation in the absence of known cause [9]. It is diagnosed by exclusion of others relevant modalities [10]. The main treatment option is conservative, with long-term laxatives. In urgent situations, such as in the prescribed case, colonoscopic decompression followed, in failed attempts, by surgical intervention. Surgical options include stoma formation or colectomy with anastomosis.

\section{References}

[1] de Betue CT, Boersma D, Oomen MW, de Jong JR. Volvulus as a complication of chronic intestinal pseudo-obstruction syndrome. Eur J Pediatr (2011) 170: 1591-1595.

[2] Antonucci A, Fronzoni L, Cogliandro L, et al. Chronic intestinal pseudo-obstruction. World J Gastroenterol 2008 May 21; 14(19): 2953-2961.

[3] Stanghellini V, Cogliandro RF, de Giorgio R, et al. Chronic intestinal pseudo-obstruction: manifestations, natural history and management. Neurogastroenterol Motil (2007) 19, 440-452.

[4] Patel RV, Njere I, Campbell A, et al. Sigmoid volvulus in an adolescent girl: staged management with emergency colonoscopic reduction and decompression followed by elective sigmoid colectomy. BMJ Case Rep 2014

[5] Lou Z, Yu EA, Zhang W, et al. Appropriate treatment of acute sigmoid volvulus in the emergency setting. World J Gastroenterol 2013 August 14; 19(30): 4979-4983.

[6] Kathrine A, Ifversen W, Kjaer DW. More patients should undergo surgery after sigmoid volvulus. World J Gastroenterol 2014 December 28; 20(48): 18384-18389.

[7] Szylberg T, Marszałek A. Diagnosis of Hirschsprung's disease with particular emphasis on histopathology. A systematic review of current literature. Prz Gastroenterol 2014; 9 (5): 264-269.

[8] de Giorgio R, Sarnelli G, Corinaldesi R, Stanghellini V. Advances in our understanding of the pathology of chronic intestinal pseudoobstruction. Gut 2004; 53: 1549-1552.

[9] Gladman MA, Scott SM, Lunniss PJ, Williams NS. Systematic review of surgical options for idiopathic megarectum and megacolon. Ann Surg 2005; 241: 562-574.

[10] Kabeer S, Dvorkin L, Carrannante J, Linehan I. A life-threatening complication of undiagnosed congenital idiopathic megacolon. BMJ Case Reports 2010. 